\title{
Precancerous lesions of cervix among women infected with HIV in Referral Hospitals of Amhara Region, Northwest Ethiopia: a cross sectional study
}

\author{
Limenih Simachew Kassa ${ }^{1}$, Worke Mulugeta Dile ${ }^{2}$, Gebresilasie Kahsay Zenebe ${ }^{3}$, Abadi Marta Berta ${ }^{3}$
}

1. Department of Midwifery, College of Health Sciences, Bahir Dar University.

2. Department of Midwifery, College of Health Sciences, Debre Tabor University.

3. Department of Midwifery, College of Medicine and Health Sciences, University of Gondar.

\begin{abstract}
Background: The risk of death from complications related to cancer of the cervix is a main health problem over the course of a woman's life in low income countries. It commonly affects women infected with HIV. Therefore, screening women infected with HIV should start in low income countries, including Ethiopia.

Methods: We conducted an institution based cross-sectional study from September $1^{\text {st }}, 2015$ to December $30^{\text {th }}$, 2015. During the study, 435 women were included in the study. The data were collected through face to face interviews and patient chart review using pre-tested and structured questionnaires. Data was also collected through visual inspection with acetic acid applied for screening and treatment. Data was entered into Epi-info version 7, cleaned and analyzed using SPSS version 20. Logistic regression analysis fitted and odds ratio with 95\% confidence interval were computed.

Results: The overall prevalence of pre-cancerous lesions of the cervix was $20.2 \%$ (95\%CI: 13\%, 29\%). Having more than one lifetime sexual partner $(\mathrm{AOR}=2.91,95 \% \mathrm{CI}: 1.13,7.52)$, a history of sexually transmitted disease (AOR=4.04, 95\%CI: 2.19, 7.44), age at first birth less than 18 years $(\mathrm{AOR}=3.36,95 \% \mathrm{CI}: 1.79,5.01)$ and baseline $\mathrm{CD} 4$ count less than 200 cells $/ \mathrm{mm}^{3}(\mathrm{AOR}=7.51$, $95 \% \mathrm{CI}: 3.58,15.68)$ were significantly associated with pre-cancerous lesion of the cervix.

Conclusion: This study points out the prevalence of pre-cancerous lesions of the cervix is high. Thus, the findings recommend raising of a screening strategy for cervical intraepithelial neoplasia for all women living with HIV should be undertaken. In addition, awareness creation about the impact of multiple sexual partner, promotion of early HIV diagnosis and timely baseline CD4 cell count is important.
\end{abstract}

Keywords: Pre-cancerous, cervical lesion, HIV, cancer, visual inspection, acetic acid.

DOI: https://dx.doi.org/10.4314/ahs.v19i1.46

Cite as: Kassa LS, Dile WM, Zenebe GK, AM B. Precancerous lesions of cervix among women infected with HIV in Referral Hospitals of Amhara Region, Northwest Ethiopia: a cross sectional study. Afri Health Sci. 2019;19(1). 1695-1704. https:/ / dx.doi. org/10.4314/ abs. v19i1.46

\section{Background}

Invasive cancer of the cervix (ICC) is a result of spread from the superficial part of the cervix to tissues deeper in the cervix or to other parts of the body. Worldwide, ICC is the third

\section{Corresponding author: \\ Limenih Simachew Kassa, Department of Midwifery, College of Health Sciences, Bahir Dar University. Email: simachewkassa@yahoo.com}

commonest cancer in women ${ }^{1,2}$. Though it is treatable, it remains a principal reason for death among women living in resource limited countries ${ }^{3}$. Worldwide, it has an occurrence rate of 371,000 cases and death rate of 190,000 with $80 \%$ of the cancer of the cervix death occurring in resource limited countries. The maximum rates are found in Central and South America, East Africa, South andSouth-East Asia and the Western Pacific. The problem is mainly worse in sub- Saharan Africa ${ }^{4}$. The age-adjusted incidence rate of cancer of the cervix in Ethiopia is 35.9 per 100,000 women. From the time of beginning of the Human Immunodeficiency Virus (HIV) epidemic, cancer of the cervix was categorized as an acquired immuno- 
deficiency syndrome (AIDS) defining cancer $^{5,6,7}$. Studies revealed that the prevalence of cytologically noticed squamous intraepithelial lesion (SIL) and infection of the cervix with human papillomavirus (HPV) are suggestively more common in women infected with HIV. Furthermore, unlike other AIDS-defining cancers, the incidence of cancer of the cervix has not reduced considerably with the growing use of antiretroviral therapy ${ }^{8-11,15}$. Therefore, the expansion of a logical method to the screening and the successive management of disease of the cervix in this part of population are essential.

Preceding practices in several Sub-Saharan countries underlined the absence of consistency of cytology based cervical screening at the population level. The reasons were inadequate access to health services and lack of screening programs ${ }^{12}$. Hence, the impacts are directly visible on the poor's, illiterates ${ }^{13}$.

Therefore, inexpensive cervical cancer screening techniques based on visual inspection of the cervix with Acetic acid (VIA) or Visual Inspection with Lugol's Iodine (VILI) have been planned and amended to resource-limited situations for years. In resource limited situations, VIA has revealed an equal strength compared to cytology. It is stated to have $80 \%$ sensitivity, $92 \%$ specificity, a $10 \%$ positive predictive value and a 99\% negative predictive value $^{14,31}$. Although screening of pre-cancerous lesion of the cervical (PCL) for women infected with HIV has been in progress in resource scarce centers in Ethiopia. Data on the prevalence and associated with the lesion is limited. Updated awareness about prevalence and associated is needed to recognize women infected with HIV who are more likely to improve pre-cancerous lesion of the cervix and to design suitable screening and treatment approaches.

\section{Methods}

\section{Study design}

An institution based cross-sectional study carried out in three referral hospitals the Amhara region of Ethiopia from September $1^{\text {st }}$ to December 30 ${ }^{\text {th }}, 2015$.

\section{Study setting}

The Amhara region is one of the nine states in Ethiopia. It is bounded by Tigray region to the North, Sudan to the West, the Afar region to the East, and the Benishangul-Gumuz to the West and SouthWest, and Oromia region to the South. Based on the 2016 Ethiopian Demo- graphic and health survey report, the prevalence of HIV in Amhara region was 2.1\%. The three referral hospitals (Debre Markos, Dessie and Felegehiwot) were included in the study. Each hospital provides care and treatment for more than 3,000 patients infected with HIV. Each referral hospital accepts an average of 80 women infected with HIV per day ${ }^{30}$.

\section{Participants}

Those HIV positive women who were visiting the ART clinic at the three hospitals and those HIV positive women who screened for pre-cancerous cervical lesion (PCL) recruited.

Women who were very ill and unable to answer question swere excluded from the study. In addition, women who had a history of diagnosed cancer of the cervix and those who had had total hysterectomy were excluded from the study.

\section{Sample size, sampling procedure and data collection}

A sample size of 435 was determined using single population proportion formula. We used the Prevalence of $22.1 \%$ pre-cancerous lesions of the cervical among women infected with HIV in Southern Ethiopia ${ }^{22}$, a confidence interval (CI) of $95 \%$, and marginal of error $4 \%$ and $5 \%$ non-response rate. Since there were only three hospitals that provide the service, all of them were selected purposively. Systematic sampling technique was employed to select each participant. Women were interviewed at health institution using pre-tested and structured questionnaires. Four trained female BSc midwives collected the data. Two MSc midwives professionals were assigned as supervisor. Both data collectors and supervisors were trained on the purpose of the study, data collection technique and tool before and after pre-test.

Trained midwives employed in the screening and treatment centres of the three hospitals conducted the screening for this study. Bi-valve speculum was placed into the vagina and the cervix visualized via a halogen focus lamp to recognize the squamo-columnar junction (SCJ).

After cleaning away any extra mucus with a cotton swab, a five percent acetic acid solution was applied to the cervix for VIA. The results were observable one minute after application.

Pre-cancerous cervical lesions were incorporated while dense aceto-white lesions with well defined margins de- 
tected in the vicinity of the transformation zone initiating from the SCJ, or if the whole cervix or cervical growth turned white. A notion of invasive cancer of the cervix was defined as any cervical ulcer or growth being observed. Results of VIA were categorized as negative, positive, or suspicious for invasive cancer of the cervix (ICC) rendering to the International Agency for Research on Cancer (IARC) training manual. Whenever there was the vagueness of the screening outcome, the midwives discussed with a trained gynaecologist and he/she would approve the finding.

\section{Operational definitions}

Negative screening test of pre-cancerous cervical lesion: No aceto white lesions on VIA which is normal cervix that remained pale and pink in colour.

Positive screening test of pre-cancerous cervical lesion: Sharp, distinct, well-defined, dense aceto white areas close to the squamo-columnar junction on VIA.

Suspicion for pre-cancerous cervical lesion: any cervical ulcer or growth being observed.

Data processing and analysis

Data was entered and analyzed using SPSS version 20. A frequency of each variable was calculated to check for outliers and missed values. Proportion of pre-cancerous lesion was determined. Logistic regression was done to analyze the data. Crude and adjusted Odds ratios were computed for each explanatory variable to determine the strength of association and control confounders. $\mathrm{P}$ value $<0.2$ was taken as a cut-off point to select variables for the multiple logistic regression models. We used backward elimination to avoid multi collinearity. A p-value of less than 0.05 was considered statistically significant.

\section{Ethical consideration}

The study was approved by the Ethical Review Committee of university of Gondar. Letter of permission was taken from respective hospitals. Written consent (finger print for those who cannot read and write) was taken from every woman. Privacy and confidentiality was maintained throughout the study period by excluding personal identifiers from the data collection form.

\section{Results}

\section{Sociodemographic characteristics}

In total, 435 women were involved in the study making a response rate of $100 \%$. The mean age of the participants was 35.9 years ( $\mathrm{SD}=4.5$ years). More than half $(53.6 \%)$ of the participants were between 30 to 39 years old. Three hundred and fifty $(80.5 \%)$ of the respondents were Orthodox Christians and the majority (97.2\%) were Amhara in Ethnicity. Three hundred seventy (85.1\%) of them were urban dwellers and 175 (40.1\%) had no formal education. More than half $(56.1 \%)$ of the participants were married and nearly a quarter of them (31\%) were housewives. Two hundred seventy-eights $(63.9 \%)$ of the women had monthly income of less than 33 USD Dollars per month per household with the median income of 28 USD Dollars (Table 1). 
Table 1: Distribution of study participants by their socio-demographic characteristic in Felege Hiwot referral hospital. Bahir dar city, NorthWest Ethiopia, September 12015 to December 30, $2015(n=265)$.

\begin{tabular}{llll}
\hline Characteristics & Frequency & Percentage & Mean(SD) \\
Age (in years) & & 12.4 & $35.6(4.2)$ \\
$20-29$ & 54 & 53.6 & \\
$30-39$ & 233 & 5.5 \\
$\geq 40$ & 148 & 56.1 \\
\hline Marital status & 24 & 22.5 \\
Single & 224 & 15.9 \\
Married & 98 & \\
Divorced & 69 & 11 \\
widowed & & 31.7 \\
\hline Occupational status & 48 & 35.6 \\
farmer & 138 & 21.6 \\
housewife & 155 & \\
Gov employee & 94 & 40.1 \\
daily laborer & & 29.7 \\
\hline Educational status & 175 & 18.9 \\
No formal education & 129 & 11.3 \\
Grade 1-8 & 82 & \\
Grade 9-12 & 49 & \\
Above 12 & & 85.1 \\
\hline Residence & & 14.9 \\
& 370 & 63.9 \\
Urban & 65 & 17.5 \\
Rural & 278 & 9.7 \\
\hline Monthly income(birr) & 76 & \\
$\leq 725$ & 39 & \\
$726-1400$ & & \\
1401-2350 & & \\
$\geq 2351$ & & \\
\hline
\end{tabular}

\section{Reproductive health characteristics}

The mean ages of menarche was $14.62(\mathrm{SD}=2.5)$ years. The mean age of first sexual intercourse was 16.77 (SD $=2.7)$ years. Likewise, the mean age at first marriage was $16.18(\mathrm{SD}=3.81)$ years and mean age at first birth was $16.77(\mathrm{SD}=8.24)$ years. Three hundred five $(71.3 \%)$ of the participants had their first sexual intercourse before the age of 18 years. More than half (51.3\%) of the study participants had their first birth before the age of 18 years. Two hundred fifty-five (58.60\%) study participants had more than one lifetime sexual partners and more than three-fourth $(87.1 \%)$ of the participants had given birth at least once with the mean birth of $2.4(\mathrm{SD}=2)$. Around one-third $(30.1 \%)$ of the participants had a history of abortion and 147 (33.8\%) participants used contraceptives during the study period. Among the contraceptive methods, $84(19.3 \%)$ used injectable contraceptive and $189(43.4 \%)$ used condom always and consistently during sexual intercourse. Nearly one-fourth (23\%) of the study participants had a history of sexually transmitted disease (STD) and $47(11.3 \%)$ of them had history of ulcerative genital lesions (Table 2). 
Table 2 Reproductive health characteristics of study participants in Felege Hiwot referral hospital. Bahir dar city, Northwest Ethiopia 2015.

\begin{tabular}{|c|c|c|c|}
\hline Characteristics & Frequency & Percentage & Mean(SD) \\
\hline Age at first sexual intercourse & & & $16.7(2.7)$ \\
\hline$<18$ & 305 & 70.1 & \\
\hline$\geq 18$ & 130 & 29.9 & \\
\hline Age at first marriage & & & $16.18(3.87)$ \\
\hline$<18$ & 267 & 61.4 & \\
\hline$\geq 18$ & 168 & 38.6 & \\
\hline Age at first birth & & & $16.77(8.24)$ \\
\hline$<18$ & 223 & 51.3 & \\
\hline$\geq 18$ & 212 & 48.7 & \\
\hline Life time number of sexual partners & & & $3.9(3.5)$ \\
\hline 1 & 75 & 17.2 & \\
\hline$\geq 2$ & 360 & 82.8 & \\
\hline Parity & & & $2.4(2)$ \\
\hline 0 & 56 & 12.9 & \\
\hline $1-4$ & 332 & 76.3 & \\
\hline$\geq 5$ & 47 & 10.8 & \\
\hline History of abortion & & & • \\
\hline yes & 131 & 30.1 & \\
\hline No & 304 & 69.9 & \\
\hline Family history of cervical cancer & & & \\
\hline Yes & 6 & 1.4 & \\
\hline No & 429 & 98.6 & \\
\hline $\begin{array}{l}\text { Use of condom during sex } \\
\text { intercourse }\end{array}$ & & & \\
\hline \multicolumn{4}{|l|}{ Yes } \\
\hline & 189 & 43.4 & \\
\hline \multicolumn{4}{|l|}{ No } \\
\hline & 246 & 56.6 & \\
\hline \multicolumn{4}{|l|}{ Ever history of STD } \\
\hline Yes & 100 & 23 & \\
\hline No & 335 & 77 & \\
\hline
\end{tabular}

Immunology, behavioral and other characteristics

Nearly all the participants $(96.6 \%)$ were currently on active anti-retroviral therapy (HAART). More than half $(58.3 \%)$ of the study participants had a baseline CD4 count of less than 200 copies $/ \mathrm{mm}^{3}$ with the mean CD4 count of 212 copies $/ \mathrm{mm}^{3}$ (SD = 133.6). Most women (91.9\%) had current CD4 counts of 200 copies $/ \mathrm{mm}^{3}$ and above with the mean CD 4 count of 479 copies $/ \mathrm{mm}^{3}$ (SD $=203.9)$. The median duration of HAART use was 36 months and $11(2.5 \%)$ participants had ever history of cigarette smoking (Table 3).

Prevalence of precancerous cervical lesion of the cervix The prevalence of pre-cancerous cervical lesion was $20.2 \% 95 \%$ CI: $13 \%, 29 \%$. 
Table 3 Immunological and behavioral characteristics of the study participants, in Felege Hiwot referral hospital. Bahir dar city, Northwest Ethiopia, $2016(n=435)$.

\begin{tabular}{llll}
\hline Characteristics & Frequency & Percentage & Mean(SD) \\
\hline Base line CD4 count & before & & \\
HAART & 253 & 58.2 & \\
$<200$ & 182 & 41.8 & $479(203.9)$ \\
$\geq 200$ & 32 & & \\
\hline Recent CD4 count after HAART & 7.4 & \\
$<200$ & 403 & 92.6 & \\
$\geq 200$ & & & \\
\hline Current use of HAART & 420 & 96.6 & \\
Yes & 15 & 3.4 & \\
No & & & \\
\hline ART clinic follow up duration & 416 & 95.6 & \\
1-6 months & 19 & 4.4 & \\
7-12months & & & \\
Current HAART use duration & 166 & 39.5 & \\
1-24 month & 111 & 26.4 & \\
25-48 month & 143 & 34 & \\
$\geq 49$ month & & & \\
\hline Ever history of smoking & 11 & 2.5 & \\
Yes & 424 & 97.5 & \\
No &
\end{tabular}

Factors associated with precancerous cervical lesion In the bivariate analysis, age, occupational status, educational status, age at first birth, history of STD, lifetime number of sexual partners, and baseline CD4 count were independently associated with pre-cancerous lesion of the cervix.

Multivariate logistic regression showed the age at first birth, number of lifetime sexual partners, history of STD, and base line CD 4 count statistically associated with pre-cancerous lesion of the cervix. Mothers whose age at first birth less than 18 years were 3.36 times more likely to develop pre-cancerous cervical lesion than those whose age at first birth more than 18 years $A O R=3.36$,
95\% CI: 1.79,6.32. Those women infected with HIV who had more than one lifetime sexual partner were 2.91 times more likely to develop pre-cancerous cervical lesion than those having one lifetime sexual partner $\mathrm{AOR}=2.91,95 \%$ CI: $1.13,7.52$.

Statistical association also depicted that those women who had a history of STD were about 4.04 times more likely to have a pre-cancerous cervical lesion than those without a history of STD AOR=4.04, 95\% CI: 2.19, 7.44. Those women whose baseline CD4 count were less than 200 cells $/ \mathrm{mm}^{3}$ were 7.51 times more likely to have precancerous cervical lesions than those patients with a baseline CD4 count above 200 cells $/ \mathrm{mm}^{3} \mathrm{AOR}=7.51,95 \%$ CI 3.58,15.68 (Table 4). 
Table 4 Logistic regression analysis of factors associated with precancerous cervical cancer lesion (PCCL) in Northwest Ethiopia, 2016.

\begin{tabular}{|c|c|c|c|c|c|}
\hline Characteristics & PCCL & & COR(95\%CI) & $\operatorname{AOR}(95 \% \mathrm{CI})$ & $\begin{array}{l}\mathbf{P}- \\
\text { value }\end{array}$ \\
\hline & Yes & No & & & \\
\hline Age & & & & & 0.32 \\
\hline $20-29$ & 8 & 48 & 1.00 & 1.00 & \\
\hline $30-39$ & 65 & 271 & $1.44(0.65,3.19)$ & $1.51(0.52,4.39)$ & \\
\hline$\geq 40$ & 15 & 28 & $3.21(1.21,8.53)$ & $2.97(0.71,10)$ & \\
\hline $\begin{array}{l}\text { Occupational } \\
\text { status }\end{array}$ & & & & & 0.06 \\
\hline Farmer & 15 & 33 & 1.00 & 1.00 & \\
\hline House wife & 30 & 108 & $0.61(0.29,1.27)$ & $0.40(0.16,1.02)$ & \\
\hline Govt employee & 32 & 123 & $0.57(0.29,1.18)$ & $0.44(0.17,1.11)$ & \\
\hline daily laborer & 11 & 83 & $0.29(0.12,0.70)$ & $0.19(0.06,0.64)$ & \\
\hline $\begin{array}{l}\text { Educational } \\
\text { status }\end{array}$ & & & & & 0.10 \\
\hline $\begin{array}{l}\text { No formal } \\
\text { education }\end{array}$ & 34 & 141 & $0.60(0.29,1.24)$ & $0.82(0.26,2.59)$ & \\
\hline Grade 1-8 & 31 & 98 & $0.79(0.38,1.66)$ & $0.97(0.32,2.92)$ & \\
\hline Grade 9-12 & 9 & 73 & $0.31(0.12,0.78)$ & $0.33(0.10,1.09)$ & \\
\hline Above 12 & 14 & 35 & 1.00 & 1.00 & \\
\hline Resident & & & & & 0.38 \\
\hline Urban & 70 & 300 & $0.609(0.33,1.11)$ & $1.57(0.57,4.44)$ & \\
\hline Rural & 18 & 47 & 1.00 & 1.00 & \\
\hline $\begin{array}{l}\text { Age at first } \\
\text { sexual } \\
\text { intercourse }\end{array}$ & & & & & 0.90 \\
\hline$<18$ & 76 & 229 & $3.26(0.99,2.52)$ & $1.05(0.50,2.19)$ & \\
\hline$\geq 18$ & 12 & 118 & 1.00 & 1.00 & \\
\hline $\begin{array}{l}\text { Age at first } \\
\text { birth }\end{array}$ & & & & & $<0.001$ \\
\hline$<18$ & 64 & 159 & $3.15(1.89,5.27)$ & $2.88(1.79,5.01)^{*}$ & \\
\hline$\geq 18$ & 24 & 188 & 1.00 & & \\
\hline$\geq 2$ & 82 & 278 & $3.39(1.42,8.09)$ & $2.91(1.13,7.52) *$ & \\
\hline $\begin{array}{l}\text { History of } \\
\text { abortion }\end{array}$ & & & & & 0.68 \\
\hline Yes & 34 & 97 & $1.62(0.99,2.65)$ & $0.86(0.41,1.73)$ & \\
\hline No & 54 & 250 & 1.00 & 1.00 & \\
\hline $\begin{array}{l}\text { Ever history of } \\
\text { STD }\end{array}$ & & & & & $<0.001$ \\
\hline Yes & 39 & 61 & $3.73(2.26,6.17)$ & $3.04(2.19,7.44) *$ & \\
\hline No & 49 & 286 & & & \\
\hline
\end{tabular}




\section{Discussion}

This study aimed to assess factors associated with precancerous cervical lesions among mothers who are HIV infected. The study shows that the overall prevalence of precancerous lesion of the cervix among women infected with HIV in Amhara regional state referral hospitals was $20.2 \%$. This study is in line with studies carried out in Ukraine $(21 \%)^{16}$ and Southern Ethiopia $(22.1 \%)^{22}$. However, it is higher than the studies carried out in Republic of Cote Devoir $(10.6 \%)^{18}$ and West Nigeria $(14.3 \%)^{19}$. The greater prevalence in this study could be due to the fact there are differences in the sexual practices of the women studied. Having multiple sexual partners because of cultural dissimilarities escalates the risk of getting HPV, and in turn, the development of cervical pre-cancer lesions. In Nigeria, where the lowest prevalence was stated, $96 \%$ of the study participants had two or less lifetime sexual partners. In the current study, the mean number of lifetime sexual partners is more than three. Also, the greater prevalence in this study could be due to the fact that there is a high chance of younger age at first sexual intercourse. The mean age of first sexual intercourse of study participants was 16.7 years, which could increase the number of life time sexual partner and risk of exposure for pre-cancerous lesion of the cervix.

This study illustrated that those mothers whose age at first birth less than 18 years were 3.36 times more likely to develop pre-cancerous lesion of the cervix compared to those whose age at first birth more than 18 years $\mathrm{AOR}=3.36,95 \% \mathrm{CI}: 1.79,6.32$. This is probably the first report of any evidence that age at first birth less than 18 years might contribute to the risk of pre-cancerous lesion of the cervix in women infected with HIV. Those women who gave birth less than 18 years would be at high exposure to HPV through early sexual intercourse and multiple sexual partners.

The present study also found that those women infected with HIV who had had more than one lifetime sexual partner were 2.91 times more likely to develop pre-cancerous lesion of the cervix than those having one lifetime sexual partner $\mathrm{AOR}=2.91,95 \% \mathrm{CI}: 1.13,7.52$. The result is similar with previous studies done in Nigeria ${ }^{26}$ , Southern Ethiopia ${ }^{22}$ and Mekelle ${ }^{27}$. The possible explanations might be those women who had more than one lifetime sexual partner could develop pre-cancerous cervical lesion because of as the number of sexual partners increases they become more prone to acquiring the HPV infection, which is the causative agent for cervical cancer.

In this study those women who had ever history of STD were about 4.04 times more likely to have a pre-cancerous lesion of the cervix than those without a history of STD $\mathrm{AOR}=4.04,95 \% \mathrm{CI}: 2.19,7.44$. This result is constant with studies conducted in Kenya ${ }^{28}$ and Southern Ethio$\mathrm{pia}^{22}$. The possible explanations might be because of the sexually transmitted nature of HPV infection. Co-infection with a STI like chlamydia trachomatis, herpes simplex or genital warts in the presence of HPV amplified the risk of CIN by causing inflammation which enables HPV tenacity and hence cervical lesion and carcinogenesis ${ }^{32}$. Finally, the current study found that participants whose baseline CD4 count less than 200cells $/ \mathrm{mm}^{3}$ were 7.51 times more likely to have precancerous cervical lesion than those whose baseline CD4 count was more than 200 cells $/ \mathrm{mm}^{3}$ AOR $=7.51,95 \%$ CI 3.58,15.68. This finding is consistent with studies done in Tanzania ${ }^{21}$ and Mekelle $^{27}$. The possible explanations might be due to the effect of immune function, HIV persuaded immunosuppression leads to incapability to control the HPV manifestation, the tenacity of HPV infection and the progress of cervical lesions.

This study didn't show any association between PCL and currently not on HAART among HIV infected women. But studies conducted in Kenya ${ }^{20}$ and Southern Ethiopia $^{22}$ stated the association between pre-cancerous lesion of the cervix and not on HAART among women infected with HIV. The possible explanation might be in Southern Ethiopia 32\% of the study participants did not start HAART but in this study majority of the study participants $(97 \%)$ started HAART. Therefore, in this study HAART might have prevented the development of precancerous and invasive cancer and caused regression of the lesion ${ }^{31}$.

\section{Limitation of the study}

This study has some inherent limitations. The first one was those mothers who were asked about the number of sexual partners might have a chance of social desirability bias. The second was the study involved only women who are infected with HIV voluntarily screened for PCL so that the generalizability of results to all women infected with HIV in Amhara region might be inadequate. Furthermore, the bias of the visual screening methods might affect the study's results in any direction. 


\section{Conclusion}

The prevalence of pre-cancerous cervical lesion among women infected with HIV in Amhara regional state referral hospitals is higher which is a similar to the situation in resource limited countries. Age at first birth less 18 years, history of lifetime STD, having more than one lifetime sexual partners were factors that were significantly associated with pre-cancerous cervical lesion. Therefore, increasing the limited screening and treatment centre is essential to escalate access of all women infected with HIV to the service. Moreover, actions intended at averting the acqusition and spread of sexully transmitted disease and decreasing the number of sexual partners are necessary.

\begin{abstract}
Abbreviations
AIDS: Acquired immune deficiency syndrome, ART: Antiretro viral therapy, CD4: Cluster of Differentiation 4, CIN:Carcinoma Insitu, HAART: Highly active antiretroviral therapy, HIV: Human immune deficiency virus, HPV: Human papilloma virus, IARC: International agency for research on cancer, ICC: Invasive cervical cancer, IRB: Institutional review board, PCL: Pre-cancerous cervical lesion, PCR: Polymerase chain reaction,PEPFAR: President's emergency plan for aids relief, SCJ: Squamo-columnar junction, SDG: Sustainable development goal, SIL: Squames intraepithelial lesion, STD: Sexual transmitted diseases,VIA: Visual inspection with acetic acid, VILI: Visual inspection with lugol's iodine.
\end{abstract}

\section{Consent for publication}

This manuscript does not contain an individual person's data, image, and video, so; consent for publication is not applicable

\section{Competing interests}

The authors declare that they have no competing interests.

\section{Funding}

The authors have no support or funding to report.

\section{Author's contribution}

Simachew Kassa wrote the proposal, took part in data collection, analyzed the data, and drafted the paper.

Mulugeta D, Kahsay Z and Marta B approved the proposal with some revisions, participated in data analysis and revised subsequent drafts of the paper. All authors read and approved the final manuscript.

\section{Acknowledgements}

We would like to thank the study participants for their participation. We were also grateful to express our gratitude to data collectors and supervisors for their time and full commitment.

\section{References}

1. World Health Organization : Comprehensive Cervical Cancer Control A guide to essential practices second edition, 2014.

2. Addis IB, KD Hatch, JS Berek, Berek \& Novak's Gynecology. $14^{\text {th }}$ Edition ed. Intraepithelial Disease of the Cervix, Vagina,and Vulva, Berek. 2007 LippincottWilliams \& Wilkins. 562.

3. Sherris, J., et al., Evidence-based, alternative cervical cancer screening approaches in low-resource settings. International Perspectives on Sexual and Reproductive Health, 2009: p. 147-152.

4. Jemal A, et al., Global cancer statistics. CA: a cancer Journal for Clinicians, 2011. 61(2):p. 69-90.

5. Control CfD and Prevention, MMWR. Recommendations and Reports-1993 Revised classification system for HIV infection and expanded surveillance case definition for AIDS among adolescents and adults, 2011, January.

6. De Vuyst H, et al. HIV, human papillomavirus, and cervical neoplasia and cancer in the era of highly active antiretroviral therapy. European Journal of Cancer Prevention, 2008. 17(6): p. 545-554.

7. World Health Organization 2014:Comprehensive Cervical Cancer Control A guide to essential practice,Geneva 27, Switzerland.Second edition ed., Geneva , Switzerland: World Health Organization

8. World Health organization: Comprehensive cancer control. A Guide to Essential Practice. World Health Organization: Geneva; 2006.

9. CSA Ethiopia and ICF International (2012) Ethiopia Demographic and Health Survey report 2011. Addis Ababa, Ethiopia and Calverton, Maryland, USA.

10. Wittet, S. and V. Tsu, Cervical cancer prevention and the Millennium Development Goals. Bulletin of the World Health Organization, 2008. 86(6): p. 488-490.

11. Sankaranarayanan R, AM Budukh, R Rajkumar. Effective screening programmes for cervical cancer in low- 
and middle-income developing countries. Bulletin of the World Health Organization, 2001. 79(10): p. 954-962.

12. Gakidou E S. Nordhagen, Z Obermeyer. Coverage of cervical cancer screening in 57 countries: low average levels and large inequalities. PLoS Medicine, 2008. 5(6): p. e132.

13. Saxenian, H., HPV vaccine adoption in developing countries: Cost and financing issues. International AIDS Vaccine Initiative (IAVI), 2007.

14. WHO /ICO (2009) Human papiloma virus and related cancers in Ethiopia. In Summary report.

15. Lehtovirta P, Paavonen J, Heikinheimo O. Risk factors, diagnosis and prognosis of cervical intraepithelial neoplasia among HIV-infected women. International journal of STD \& AIDS. 2008 Jan; 19(1): 37-41.

16. Bailey $\mathrm{H}$ et al., Cervical screening within HIV care: findings from an HIV-positive cohort in Ukraine. PLoS One, 2012. 7(4): p. e34706.

17. Sahasrabuddhe, VV et al., Prevalence and predictors of colposcopic-histopathologically confirmed cervical intraepithelial neoplasia in HIV-infected women in India. PLoS One, 2010. 5(1): p. e8634.

18. Jaquet A et al., Cervical human papillomavirus and HIV infection in women of child-bearing age in Abidjan, Cote d'Ivoire, 2010. Br J Cancer, 2012. 107(3): p. 556-63.

19. Ezechi OC et al., The association between HIV infection, antiretroviral therapy and cervical squamous intraepithelial lesions in South Western Nigerian women. PLoS One, 2014. 9(5): p. e97150.

20. Parham GP et al., Prevalence and predictors of squamous intraepithelial lesions of the cervix in HIV-infected women in Lusaka, Zambia. Gynecol Oncol, 2006. 103(3): p. 1017-22.

21. Kahesa $C$ et al., Association between invasive cancer of the cervix and HIV-1 infection in Tanzania: the need for dual screening. BMC Public Health, 2008. 8: p. 262.

22. Gedefaw AA Astatkie, GA Tessema. The prevalence of precancerous cervical cancer lesion among HIV-in- fected women in southern Ethiopia: a cross-sectional study. PLoS One, 2013. 8(12): p. e84519.

23. Kafuruki L et al., Prevalence and predictors of Cervical Intraepithelial Neoplasia among HIV infected women at Bugando Medical Centre, Mwanza-Tanzania. Infect Agent Cancer, 2013. 8(1): p. 45.

24. de Andrade AC et al., Factors associated with colposcopy-histopathology confirmed cervical intraepithelial neoplasia among HIV-infected women from Rio De Janeiro, Brazil. PLoS One, 2011. 6(3): p. e18297.

25. Moodley JR et al., HIV and pre-neoplastic and neoplastic lesions of the cervix in South Africa: a case-control study. BMC Cancer, 2006. 6: p. 135.

26. Ononogbu U et al. Cervical cancer risk factors among HIV-infected Nigerian women. BMC Public Health, 2013. 13: p. 582.

27. Gessesse $Z$ et al. Determinant factors of Visual Inspection with Acetic Acid (VIA) positive lesions among HIV positive women in Mekelle Hospital, Northern Ethiopia: A case control study. EMJ, 2015.

28. Keller, MJ et al. Risk of cervical precancer and cancer among HIV-infected women with normal cervical cytology and no evidence of oncogenic HPV infection. JAMA, 2012. 308(4): p. 362-9.

29. Memiah P et al. Prevalence and Risk Factors Associated with Precancerous Cervical Cancer Lesions among HIV-Infected Women in Resource-Limited Settings. AIDS Research and Treatment, 2012. 2012: p. 1-7.

30. Amhara regional state, Amhara region health bureau report 2013.

31. Alliance for Cervical Cancer Prevention (ACCP) \& World Health Organization. Cervical cancer prevention in developing countries: A review of screening and programmatic strategies. (Forthcoming, November 2003). 32. Mbulaiteye SM et al. HIV and cancer in Africa: mutual collaboration between HIV and cancer programs may provide timely research and public health data. Infect Agent Cancer, 2011. 6(1): p. 16. 